\title{
Epidural hematoma following spinal cord stimulator implant
}

\author{
Brian T. Kloss • Anne Marie Sullivan • Elliot Rodriguez
}

Received: 23 December 2009 / Accepted: 25 February 2010/Published online: 18 May 2010

(C) Springer-Verlag London Ltd 2010

\begin{abstract}
A 50-year-old male with chronic low-back pain presented to the ED with mid-back pain, numbness, paresthesia and weakness in both legs. These symptoms were preceded by a sudden onset of paralysis below the waistline without loss of bowel or bladder control, which occurred during the surgical placement of a neurostimulator. The device was immediately removed, and the patient's paralysis partially resolved. He transitioned into his current symptom complex. He was immediately transferred to the ED for evaluation. Physical exam showed stable vital signs, tenderness to palpation in the spine, decreased sensation T12 and below, 4/5 strength in the lower extremities, and $2+$ distal pulses, intact perianal sensation but decreased rectal tone. MRIs of the thoracic and lumbar spines were obtained, revealing an epidural hematoma from T8-L2.

The MRI results shown in Fig. 1 display abnormal signal posterior to the spinal cord (A) that is predominantly hyperintense to the spinal cord on this T2-weighted image. There is compression of the cord at the T8 vertebral body
\end{abstract}

B. T. Kloss $(\bowtie) \cdot$ E. Rodriguez

SUNY Upstate Medical University,

Department of Emergency Medicine,

550 E Genesee St,

Syracuse, NY 13202, USA

e-mail: klossb@upstate.edu

E. Rodriguez

e-mail: rodrigue@upstate.edu

A. M. Sullivan

SUNY Upstate Medical University,

Department of Radiology,

750 East Adams Street,

Syracuse, NY 13210-2342, USA

e-mail: adena@upstate.edu level (A). No abnormal signal is seen within the cord itself. Epidural fat (B) and CSF (C) reveal that the hematoma is in the epidural space displacing the dura anteriorly. Figure 2 is a T1-weighted image revealing an abnormal signal (D) that is predominantly isointense to the spinal cord, again demonstrating compression of the cord. Lastly, Fig. 3 shows an axial T1-weighted image that shows abnormal signal within the spinal canal (E) compressing the spinal cord $(*)$.

The patient underwent an emergent hematoma evacuation and concurrent T6-L1 laminectomy with in situ fusion. He tolerated the procedure well and was discharged 6 days later to a rehabilitation facility with some improvement to

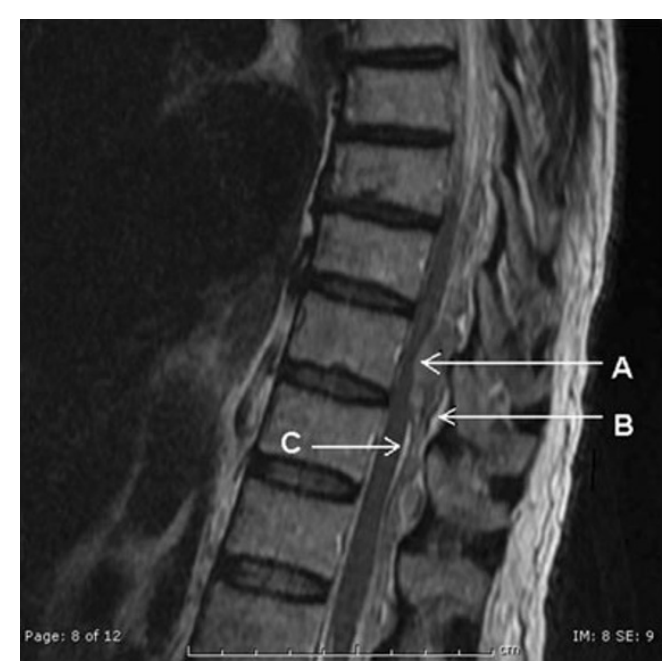

Fig. 1 T2-weighted MRI in the sagittal view. Abnormal signal posterior to the spinal cord (A) is predominantly hyperintense to the spinal cord on this T2-weighted image. There is compression of the cord at the T8 vertebral body level (A). No abnormal signal is seen within the cord itself. Epidural fat (B) and CSF (C) reveal that the hematoma is in the epidural space displacing the dura anteriorly 


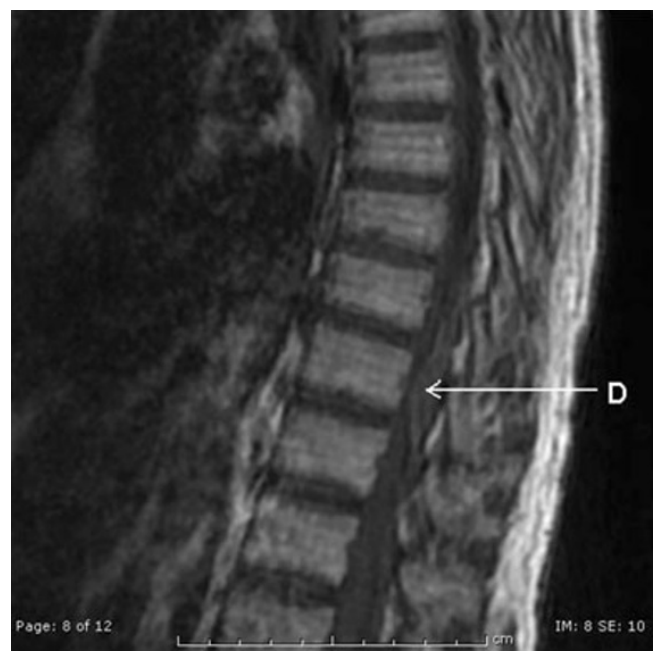

Fig. 2 T1-weighted MRI in the sagittal view. Abnormal signal (D) is predominantly isointense to the spinal cord, again demonstrating compression of the cord

his lower extremity strength, sensation and bladder and rectal control.

Epidural hematoma formation after placement of an epidural spinal cord electrode is an extremely rare and serious complication. Our PubMed search only yielded two similar cases in the published literature, neither of which was in English [1,2].

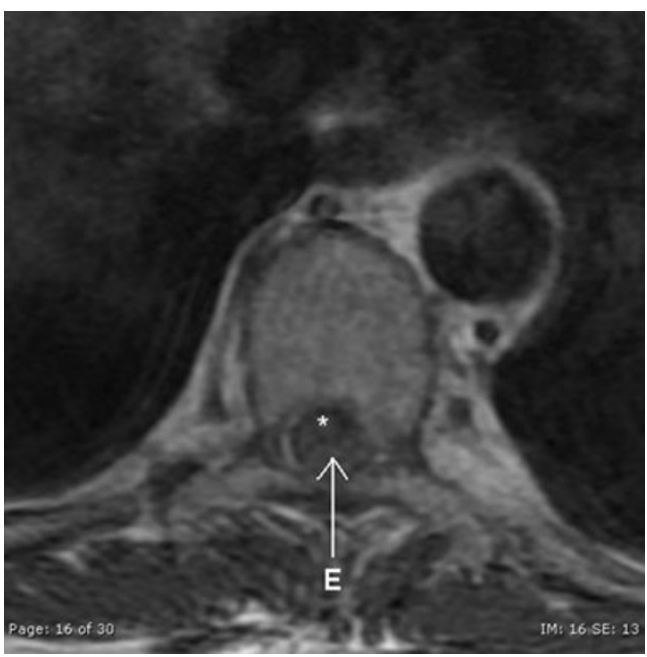

Fig. 3 T1-weighted MRI cross-sectional view. Abnormal signal within the spinal canal (E) compresses the spinal cord $(*)$

\section{References}

1. Franzini A, Ferroli P, Marras C, Broggi G (2005) Huge epidural hematoma after surgery for spinal cord stimulation. Acta Neurochir (Wien) 147(5):565-567

2. Santiago FM, Santiago J, Prieto M et al (2005) Dorsal epidural hematoma after implantation of a dorsal nerve stimulator. Rev Esp Anestesiol Reanim 52(7):440-441 